\title{
CONSULTING FORESTERS IN CANADA
}

The following list of Consulting Foresters is published by the Canadian Institute of Forestry. Individuals listed are members of the C.I.F. who have answered the questionnaire sent to all members in April, 1959.

Listing is in alphabetical order and by province.

AYERS, M. J.

BAXTER, D. H.

BUCKLAND, A. C.

CAESAR, Robin L.

COLLINS, J. R.

DEGRACE, L. A.

\section{BRITISH COLUMBIA}

1775 Broadmead Ave., Victoria, B.C.

Full-time

Specialization: Forest Management

(Surveys, Timber, Cruising, Logging, Timberland Evaluation)

BAKEWELL, David R. Consolidated Services Limited, 325 Howe Street, Vancouver, B.C.

Full-time

Specialization: Forest Engineering, Logging \& Forest Management

Clearwater, B.C.

Part-time

Specialization: Logging Plans, Forest Surveys \& Cruises, Management Working Plans

1015 Aubeneau Crescent, West Vancouver, B.C. Full-time

Specialization: Road Locations \& Logging Plans

Consolidated Services Ltd., 325 Howe Street, Vancouver 1, B.C.

Full-time

Specialization: Forest Inventory, Forest Management

1409 W. Pender Street, Vancouver, B.C. Full-time

Specialization: General Forestry

Industrial Forestry Service Ltd., 1591 Fourth Ave., Prince George, B.C.

Full-time

Specialization: General Forestry, Timber Management

GAIRNS, Charles Henry Industrial Forestry Service Ltd., 1591 Fourth Avenue, Prince George, B.C. Full-time

Specialization: Cruising, Engineering and General Logging Planning, particularly in northern B.C. 
GORMELY, M. W.

JOHNSON, F. J. G.

KER, John W.

MACQUEEN, I. C.

MINNS, G. W.

NOVAK, Nicholas

ORCHARD, C. D.
FORESTRY CHRONICLE

413 Dominion Building, 207 West Hastings St., (Opposite Victory Square), Vancouver 3, B.C.

Full-time

Specialization: Management, Logging Engineering Protection, Inventory \& other surveys, Economics, Appraisals Photogrammetry, General Practice, etc.

Suite 605, 325 Howe Street, Vancouver 1, B.C. Full-time

Specialization: Management (Timber \& Forest) Engineering (Logging, Sawmilling Utilization, Research \& Surveys) Finances (Banking, Taxation, Contracts, Legislation, etc.)

4875 College Highroad, Vancouver 8, B.C. Part-time

Specialization: Forest Measurements and Forest Economics

1409 West Pender St., Vancouver, B.C.

Full-time

Specialization: Forest Management, Industrial Log Production

McCANNEL, Kenneth C. 709 Metropolitan Bldg., 837 West Hastings St., Vancouver 1, B.C.

Full-time

Specialization: Consultations, Industrial Development and Negotiations

MILNER, Laurence J. Box 675, Quesnel, B.C.

Full-time

Specialization: Timber Sale Management in B.C. Road Location, Cruising \&

Logging Plans

Oliver Sawmills, Oliver, B.C.

Full-time

Specialization: Layouts of sustained yield units 6568 Lambert Crescent; R.R. \#1, North Surrey, B.C.

Full-time

Specialization: Logging Plans, Cruising \& Appraisals, Aerial Photographic Mapping, Tree Farm Licences

2331 Lansdowne Rd., Victoria, B.C.

Full-time

Specialization: Forest Administration 
PAINTER, M. F.

M. F. Painter \& Associates, 4607 Connaught Drive, Vancouver 9, B.C.

Full-time

Specialization: Forest Engineering

RAINSFORD, Frank G. 923 Fort St., Victoria, B.C.

Full-time

Specialization: Land \& Timber Appraisal, Forest Land Taxation \& Licensed Agent

REID, E. S.

Director, Forestal, Forestry \& Engineering International Ltd., 1409 West Pender St., Vancouver, B.C.

Full-time

Specialization: Photogrammetry as applied to Forest Surveys

RICHMOND, Hector A. Lofthouse Road, R.R. \#2, Nanaimo, B.C. Full-time

Specialization: Forest Entomology, Insect Control, Damage Appraisals, Insect Surveys, etc.

ROSS, Douglas $\mathrm{H}$.

Box 462, Vernon, B.C.

Full-time

Specialization: Timber Cruising and Forest Management

SAVOLA, R. A.

413 Dominion Building, 207 West Hastings Street, (Opposite Victory Square), Vancouver 3 B.C.

Full-time

Specialization: Management, Logging Engineering, Protection, Inventory \& other Surveys, Economics, Appraisals Photogrammetry, General Practice, etc.

SCHIEDEL, Ian

$609-837$ W. Hastings, Vancouver, B.C.

$12084-14$ th Ave., Haney, B.C.

Full-time

Specialization: B.C. Logging Layouts, Inventories \& Timber Management

SCHULTZ, C. D.

C. D. Schultz \& Company Limited, 325 Howe Street, Vancouver, B.C.

Full-time

Specialization: Timber Development, Timber Management, Wood handling methods, Analysis and control 
SCHULTZ, Robert

SMITH, D. W.

SMITH, Ralph E.

ST. CLAIR, R. C.
Robert Schultz \& Company, 425 Howe Street, Vancouver 1, B.C.

Full-time

Specialization: Forest Management \& Utilization

D. W. Smith Ltd, 625 Fort St., Victoria, B.C.

Full-time

Specialization: "Timber Management Services"

1304 Burrard Bldg., Vancouver 5, B.C.

Part-time

Specialization: Pacific Coast, Marketing all

Forest Products Transportation

R. C. St. Clair \& Associates,

608-837 West Hastings St., Vancouver, B.C.

Full-time

Specialization: Cost \& Economic Studies, Management \& Silviculture, Forest Protection, Taxation, Surveying \& Mapping, Timber Appraisal \& Valuation, Timber Volume and Quantity Estimates

VAN DOORNINCK, W. B. 413 Dominion Building, 207 West Hastings St. (Opposite Victory Square), Vancouver 3, B.C.

Full-time

Specialization: Management, Logging Engineering, Protection, Inventory \& other Surveys, Economics, Appraisals, Photogrammetry, General Practice, etc.

WALKER, Cedric W.

Swan Wooster Engineering Co., 1334 West Pender St., Vancouver, B.C.

Full-time

Specialization: Timber Management, Forest Surveys, Inventories, Valuations

WELLWOOD, R. W.

Faculty of Forestry, University of British Columbia, Vancouver 8, B.C.

Part-time

Specialization: Wood Technology, Forest Products, Utilization

\section{ONTARIO}

ANDREWS, J. R. T. Hunting Technical \& Exploration Services Ltd. 1450 O'Conner Drive, Toronto, Ontario Full-time

Specialization: Management Plans 
ATWOOD, C. E.

BROWN, W. G. E.
Department of Zoology, University of Toronto Toronto, Ontario

Part-time

Specialization: Forest Insects

Spartan Air Services, 2117 Carling Ave.

Ottawa, Ontario

Full-time

Specialization: Management \& Operational Inventory Plans, Soils (Engineering \& Ecological), Mapping, Road \& R.R. Location, Photography

CARLISLE, Kenneth W. Box 394, Iroquois Falls, Ontario

Part-time

Specialization: Mechanization and Logging Engineering

COKE, R. E.

DAY, B. G.

DELAHEY, Wallace A.

HARRIS, W. G.

LOCKHART, D. D.

MARRITT, I. C.

JECKALEJS, $\mathrm{H}$.

McCORMACK, R. J.
20 Berkinshaw Crescent Don Mills, Ontario

Part-time

Specialization: Christmas tree farms

437 Patricia Blvd., Timmins, Ontario

Full-time

Specialization: Logging, Utilization, Woodlot Management

284 Glencairn Ave., Toronto 12, Ontario

Part-time

Specialization: Forest Management and Logging

Nipigon, Ontario

Part-time

Specialization: Plywood Feasibility Studies

13 Suburban Drive, Streetsville, Ontario

Part-time

Specialization: Woodlot Management, Watershed Control

Galt, Ontario (R.R. \#4)

Full-time

Specialization: Woodlot Management, Reforestation, Christmas Tree Culture

178 Ranleigh Ave., Toronto 12, Ontario

Part-time

Specialization: Reforestation

Hunting Technical \& Exploration Services Ltd. 1450 O'Conner Drive, Toronto, Ontario

Full-time

Specialization: Site \& Soil Investigations
R.R. \#2, Rockwood, Ontario 
McGEE, C. J.

MERRITT, Victor G.

NIX, L. A.

PARSONS, H. H.

TOMASZEWSKI, Felix

WILDE, C. J. R.

MURPHY, R. J. K.
Box 692, 174 Larch St., Sudbury, Ontario Full-time

Specialization: Forest Management and Operating Plans, Wood and Timber Limit Procurement for clients

Hunting Technical \& Exploration Services Ltd. 1450 O'Conner Drive, Toronto, Ontario Full-time

Specialization: Forest Survey \& Inventories, Temperate \& Tropical, Aerial Photography

Box 804, Aylmer, Ontario

Part-time

Specialization: Woodland Valuation, in Southwestern Ontario

260 Main St. West, Brampton, Ontario Full-time

Specialization: Forest Management \& Logging

17 Black Dons Crescent, Islington, Ontario Full-time

Specialization: Aerial Timber Sketching, Aerial Photo Typing, Inventories, Management Operating Plans

451 Churchill Ave., Ottawa 3, Ontario (Apt. 6) Part-time

Specialization: Inventory, Compilation of Management and Operating Plans, Maps

357 Batsford St., Newmarket, Ontario Full-time

Specialization: Forest Engineering (logging, road construction, road location), Forest Mensuration-Photo Interpretation-Inventories-Surveys

\section{QUEBEC}

500 Tasse, Montreal 9, Quebec Full-time

Specialization: Logging Engineering

BOIVIN, Yves
1191 12th Avenue, Grand-Mere, Quebec Full-time

Specialization: Forestry, Land Surveying (in Quebec only) 
BOURGET, Marie-Albert BOURGE, Marie-Albert

BRULE, J. D.

EMOND, Laval

GOBEIL, A. R.

JENKINS, Frank T.

JONES, A. R. C.

GREGOIRE, L. J.
86 Cote de la Montagne, Quebec, Quebec Full-time

Specialization: Setting \& Drawing of Boundary Lines, Timber Limits, Mining Grounds, Town Planning, Urban and Rural Developments, Valuation, Working \& Logging Plans of Forest Properties

Val-Brillant Co., Matapedia, Quebec Part-time

Specialization: Forestry Surveys, Forest Fire Protection, Silviculture, Appraising of Timberlands

1321 Oak Avenue, Sillery, Quebec Part-time

Specialization: Secondary Power Transmission Lines

100 d'Youville, Quebec 4, Quebec

Full-time

Specialization: Inventories, Management Plans, Timberlands Brokerage and Negotiations, Public Relations

Kingsmere, Quebec

Full-time

Specialization: Forest Surveys, Forest Management Plans, Photo Interpretations, Aerial Surveys and Reconnaissance, Sketching

P.O. Box 202, Hull, Quebec

Full-time

Specialization: Forest or Timber Management

Plans, Interpretation and Map Compilation from aerial photographs, Land Surveys, Forest Surveys

Box 162, Macdonald College, Quebec Part-time

Specialization: Woodlot Advisory Work, Forest Inventory, Management Plans, Timber Marking \& Marketing

675 St. Amable St., Quebec, Quebec Part-time

Specialization: Forest Economics 
MILOT, Alfred

COOK, Chester F.

GIBSON, J. Miles

SEHEULT, L. R.

FELLOWS, E. S.
57-59 Rochette Ave., Quebec 10, Quebec Full-time

Specialization: Consultation, Inventories and Working Plans, Interpretation of aerial photographs for forestry and forest operations purposes

\section{NEW BRUNSWICK}

R.R. \#1, Fredericton, New Brunswick Full-time

Specialization: Inventory and Management Surveys-Road Locations

P.O. Box 354, Fredericton, New Brunswick Full-time

Specialization: Industry development, Forest Products, Utilization Problems, Forest Economics, Forest Administration, Forest Policy

161 Winslow St., Fredericton, New Brunswick Part-time

Specialization: Forest Management

Faculty of Forestry, University of New Brunswick, Fredericton, New Brunswick Part-time

Specialization: Logging Engineering

\section{NOVA SCOTIA}

KINGSLAND, Lloyd F. 13 Roosevelt Avenue, Truro, Nova Scotia Part-time Specialization: Wood Preservation

WRIGHT, L. LeRoy

61 Rivers Street, Kentville, Nova Scotia Part-time

Specialization: Balsam Fir, Christmas Tree Growing

U. S. A.

COOLIDGE, P. T.

Box 102, Bangor, Maine (Retired)

MOIR, Stuart
Diamond Head Road, Oswego, Oregon, 